\title{
Comment On: Sarcopenia is a Prognostic Outcome Marker in Children with High-Risk Hepatoblastoma Sarcopenia in Children? A Repechage or 'Better Late Than Never'
}

\author{
Sarah Razaq ${ }^{1}$, Murat Kara ${ }^{2}$, and Levent Özcakar ${ }^{2}$ \\ ${ }^{1}$ Combined Military Hospital, Quetta, Pakistan \\ ${ }^{2}$ Hacettepe University Faculty of Medicine
}

January 23, 2021

Comment On: Sarcopenia is a Prognostic Outcome Marker in Children with High-Risk Hepatoblastoma

\author{
Sarcopenia in Children? A Repechage or 'Better Late Than Never' \\ Sarah Razaq, FCPS ${ }^{1}$, Murat Kara, MD², Levent Özcakar MD, Professor ${ }^{2}$ \\ ${ }^{1}$ Department of Physical medicine \& rehabilitation, Combined Military Hospital, Quetta, Pakistan \\ ${ }^{2}$ Hacettepe University Medical School, Department of Physical and Rehabilitation Medicien, Ankara, Turkey \\ * Correspondence to:
}

Sarah Razaq, FCPS, Department of physical medicine \& Rehabilitation, Combined Military Hospital, Quetta, 87300, Pakistan

Tel.: 0092-3336123022, Email: docsrazaq@gmail.com

Text word count 529;

Abstract word count: Not included;

Brief running title: Sarcopenia in children

Key words: Sarcopenia, children, Muscle mass

Tables: Nil

Figures: Nil

Dear Editor,

With much interest, we have gone through the recently published article (Sarcopenia is a prognostic outcome marker in children with high-risk hepatoblastoma) by Ritz et al. ${ }^{1}$. We wish to congratulate the authors for measuring total psoas muscle area as a prognostic risk factor in children with hepatoblastoma. They have measured the cross-sectional muscle area at two distinct lumbar levels (L3-4 and L4-5) on computed tomography or magnetic resonance imaging using a freely-available online $z$-score calculator tool. ${ }^{2}$

Herewith, our point of concern is two-fold; we would like to comment on the use of the term 'sarcopenia' in children and on the lack of functional evaluation. Regarding the former, it is well-known that sarcopenia is age-related loss of muscle mass and muscle function, which ensues as early as in $5^{\text {th }}$ decade (at middle 
age) but not in children or young adults. Therefore, we need to note that there is a misunderstanding as regards the definition. Sarcopenia was first described in 1989 as an age-related decline in lean body mass affecting mobility, nutritional status, and independence. ${ }^{3}$ Of note, it is not muscle wasting or atrophy (due to immobilization, malnutrition, infection, cachexia or any adverse treatment effect) alone and should be accompanied by loss of muscle function as well. Herein, muscle function progressively deteriorates due to motoneuron loss that is not adequately compensated by reinnervation of muscle fibers by the remaining motoneurons. ${ }^{4}$ The aging-related denervation/reinnervation process has a strong impact on the quantitative changes in muscle (e.g. muscle fiber loss and atrophy), resulting in overall loss of muscle mass and muscle function. ${ }^{4}$

Second, the reduced muscle mass in the absence of functional evaluation can/should not be labelled as sarcopenia. Although for decades, this term has been used to describe the loss of muscle mass alone without reference to function, and is still being used in some cancer and other disease-related sarcopenia studies; ${ }^{5}$ after 2010, a big milestone i.e. muscle function (loss) was introduced into the concept/definition of sarcopenia. ${ }^{6}$ This new focus on muscle function is usually defined by muscle strength and power (or physical performance) and these parameters were shown to be more powerful predictors of clinical outcomes than muscle mass alone. ${ }^{7}$ Hence, similar to adults, performance evaluation can possibly be carried out via measuring grip strength (for muscle function) and anterior thigh muscle thickness (for muscle mass) in children as well. ${ }^{8}$ Of additional note, the other important milestone has been the recognition of "age-related sarcopenia (M62.84)" as an independent condition i.e. different from "muscle wasting and atrophy, not elsewhere classified (M62.5)" according to the International Classification of Diseases-10 code in $2016 .^{9}$

Lastly, muscle loss due to aging (i.e. sarcopenia), immobilization or malnutrition (i.e. muscle wasting and atrophy) is not uniform throughout the body, and it is noteworthy that anterior thigh muscles (rich in type 2 fibers) undergo atrophy earlier than the other muscle groups. ${ }^{8}$ Therefore, aside from promptly defining the pertinent clinical scenarios, we emphasize the use of basic but vital functional test (i.e. grip strength) together with the anterior thigh muscle thickness measurements (by ultrasound) in their assessments. This way, loss of muscle mass and function as well as the overall health status can accurately be managed in children. ${ }^{10}$

\section{References}

1. Ritz A, Kolorz J, Hubertus J, Ley-Zaporozhan J, Schweinitz DV, Koletzko S, et al. Sarcopenia is a prognostic outcome marker in children with high-risk hepatoblastoma. Pediatr Blood Cancer 2021; 28862 .

2. Lurz E, Patel H, Lebovic G, et al. Paediatric reference values for total psoas muscle area. J Cachexia Sarcopenia Muscle 2020;11:405-14.

3. Rosenberg IH. Sarcopenia: origins and clinical relevance. J Nutr 1997;127: 990S-991S.

4. Larsson L, Degens H, Li M, Salviati L, et al. Sarcopenia: Aging-related loss of muscle mass and function. Physiol Rev 2019;99:427-511.

5. Cruz-Jentoft AJ, Sayer AA. Sarcopenia. Lancet 2019;393:2636-46.

6. Cruz-Jentoft AJ, Baeyens JP, Bauer JM, et al. Sarcopenia: European consensus on definition and diagnosis: report of the European Working Group on sarcopenia in older people. Age Ageing 2010;39:412-23.

7. Visser M, Schaap LA. Consequences of sarcopenia. Clin Geriatr Med 2011;27:387-99.

8. Kara M, Kaymak B, Ata AM, et al. STAR - Sonographic thigh adjustment ratio: A Golden formula for the diagnosis of sarcopenia. Am J Phys Med Rehabil 2020;99:902-8.

9. Anker SD, Morley JE, von Haehling S. Welcome to the ICD-10 code for sarcopenia. J Cachexia Sarcopenia Muscle 2016;7:512-14.

10. Wind AE, Takken T, Helders PJ, Engelbert RH. Is grip strength a predictor for total muscle strength in healthy children, adolescents, and young adults? Eur J Pediatr. 2010;169:281-7. 